\title{
Generalized Water-Processed Metal Chalcogenide Complexes: Synthesis and Applications
}

Zhe Xia, ${ }^{\dagger}$ Jie Zhong, ${ }^{\dagger}$ Meiying Leng, ${ }^{\dagger}$ Long Hu, ${ }^{\dagger}$ Ding-Jiang Xue,,${ }^{, \dagger}$ Bo Yang, ${ }^{\dagger}$ Ying Zhou, ${ }^{\dagger}$ Xinsheng Liu, ${ }^{\dagger}$ Sikai Qin, ${ }^{\dagger}$ Yi-Bing Cheng, ${ }^{\dagger, \dagger}$ and Jiang Tang ${ }^{*, \dagger}$

${ }^{\dagger}$ Wuhan National Laboratory for Optoelectronics (WNLO) and School of Optical and Electronic Information, Huazhong University of Science and Technology (HUST), Wuhan, 430074, China

${ }^{\ddagger}$ Department of Materials Engineering, Monash University, VIC 380o, Australia

* To whom correspondence should be addressed. E-mail: djxue@iccas.ac.cn; jtang@email.hust.edu.cn

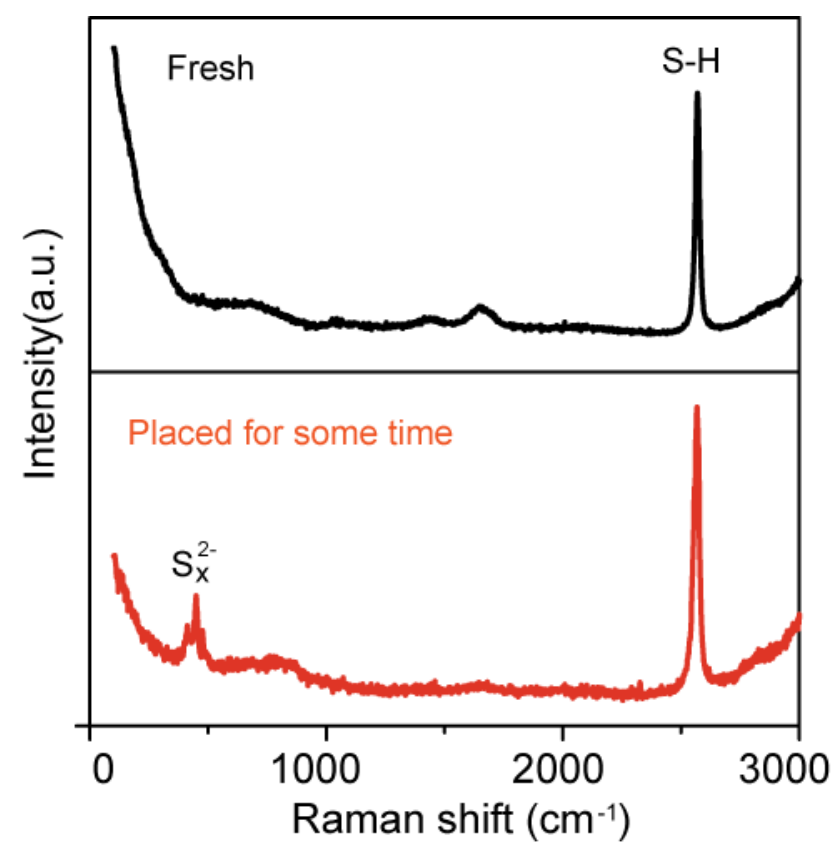

Figure S1. Raman spectra of aqueous $\left(\mathrm{NH}_{4}\right)_{2} \mathrm{~S}$ solution. 

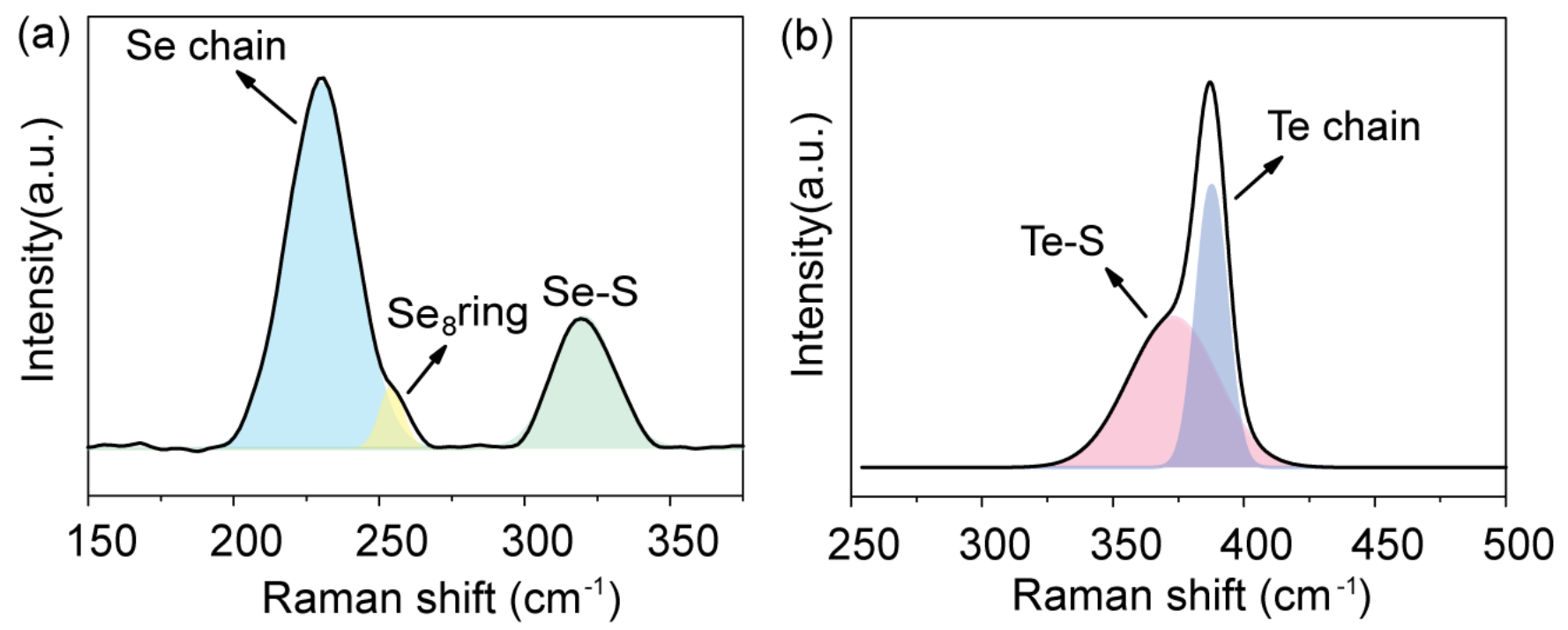

Figure S2. Raman spectra of (a) solution of Se in aqueous $\left(\mathrm{NH}_{4}\right)_{2} \mathrm{~S}$ solution, and (b) solution of Te in aqueous $\left(\mathrm{NH}_{4}\right)_{2} \mathrm{~S}$ solution.
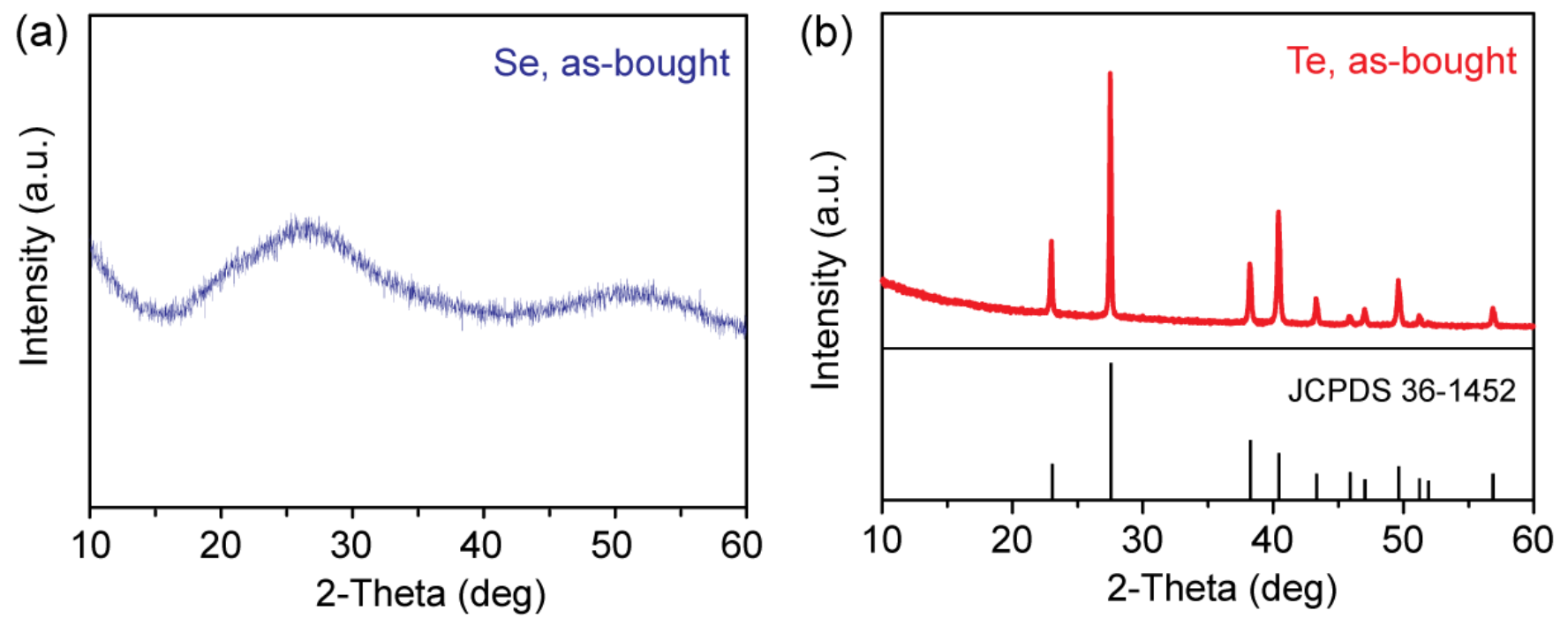

Figure S3. XRD patterns of (a) as-bought Se (99.999\%, Alfa Assar) and (b) as-bought Te (99.99\%, Aladdin). 

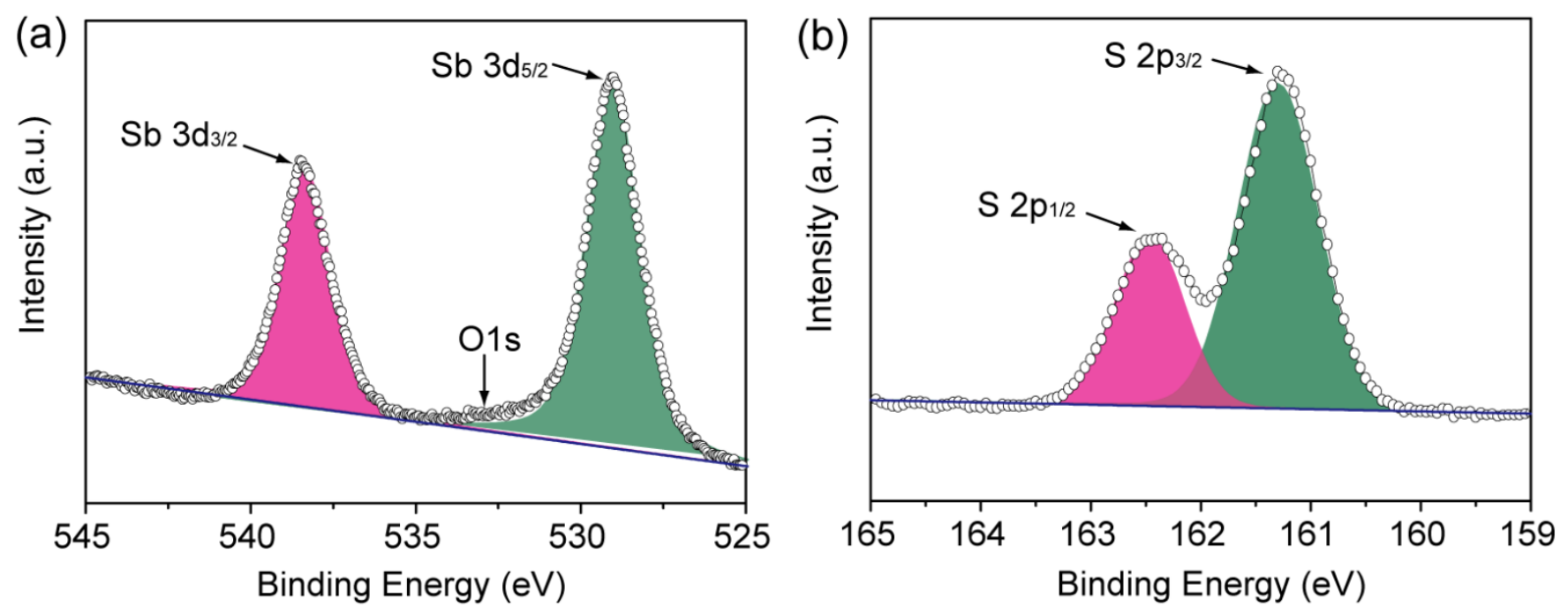

Figure $\mathrm{S}_{4}$. XPS spectra of $\mathrm{Sb}_{2} \mathrm{~S}_{3}$ prepared through thermal decomposition of $\mathrm{Sb}_{2} \mathrm{~S}_{3}-\mathrm{MCC}$ at 300 ${ }^{\circ} \mathrm{C}$ : (a) Sb $3 \mathrm{~d}$ and (b) $\mathrm{S} 2 \mathrm{p}$. Obviously, the $\mathrm{O}$ is peak is not detected near the $533 \mathrm{eV}$, indicating the oxygen concentration is too low to be detected by XPS.
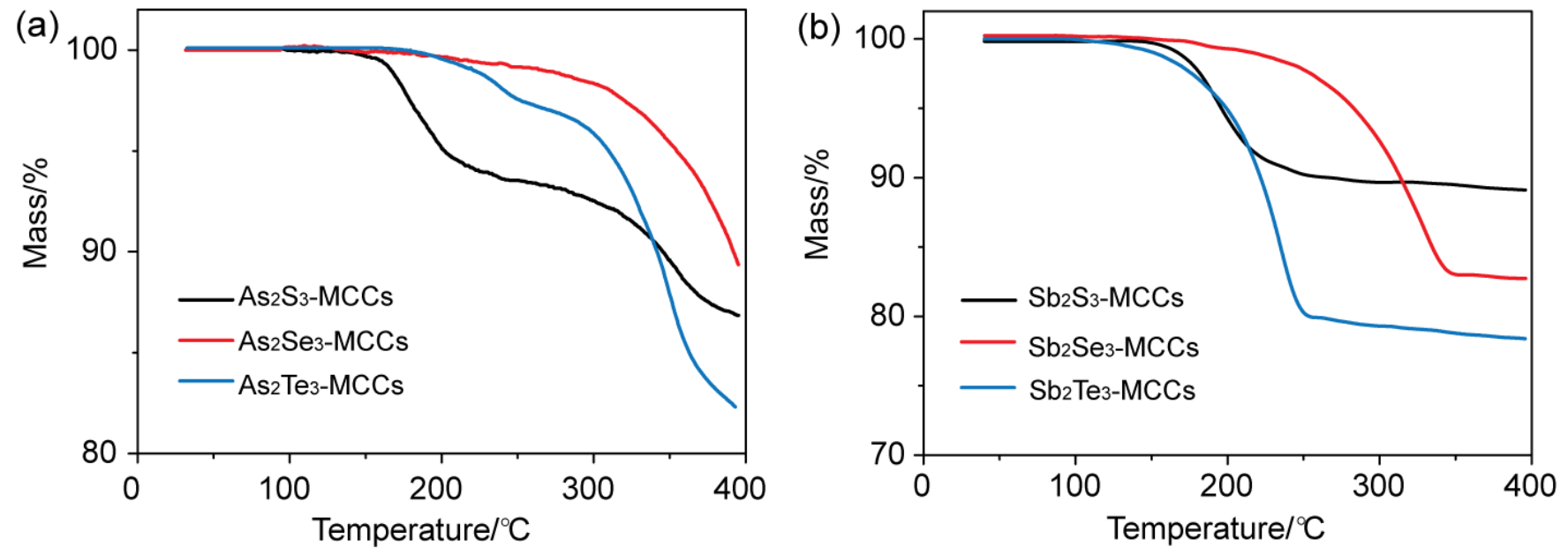

Figure $\mathrm{S}_{5}$. (a) TGA of $\mathrm{As}_{2} \mathrm{~S}_{3}-\mathrm{MCCs}, \mathrm{As}_{2} \mathrm{Se}_{3}-\mathrm{MCCs}$, and $\mathrm{As}_{2} \mathrm{Te}_{3}$-MCCs. (b) TGA of $\mathrm{Sb}_{2} \mathrm{~S}_{3}-\mathrm{MCC}$, $\mathrm{Sb}_{2} \mathrm{Se}_{3}-\mathrm{MCCs}$, and $\mathrm{Sb}_{2} \mathrm{Te}_{3}-\mathrm{MCCs}$ (run at $10{ }^{\circ} \mathrm{C} \mathrm{min}^{-1}$ in a $\mathrm{N}_{2}$ flowing environment). 

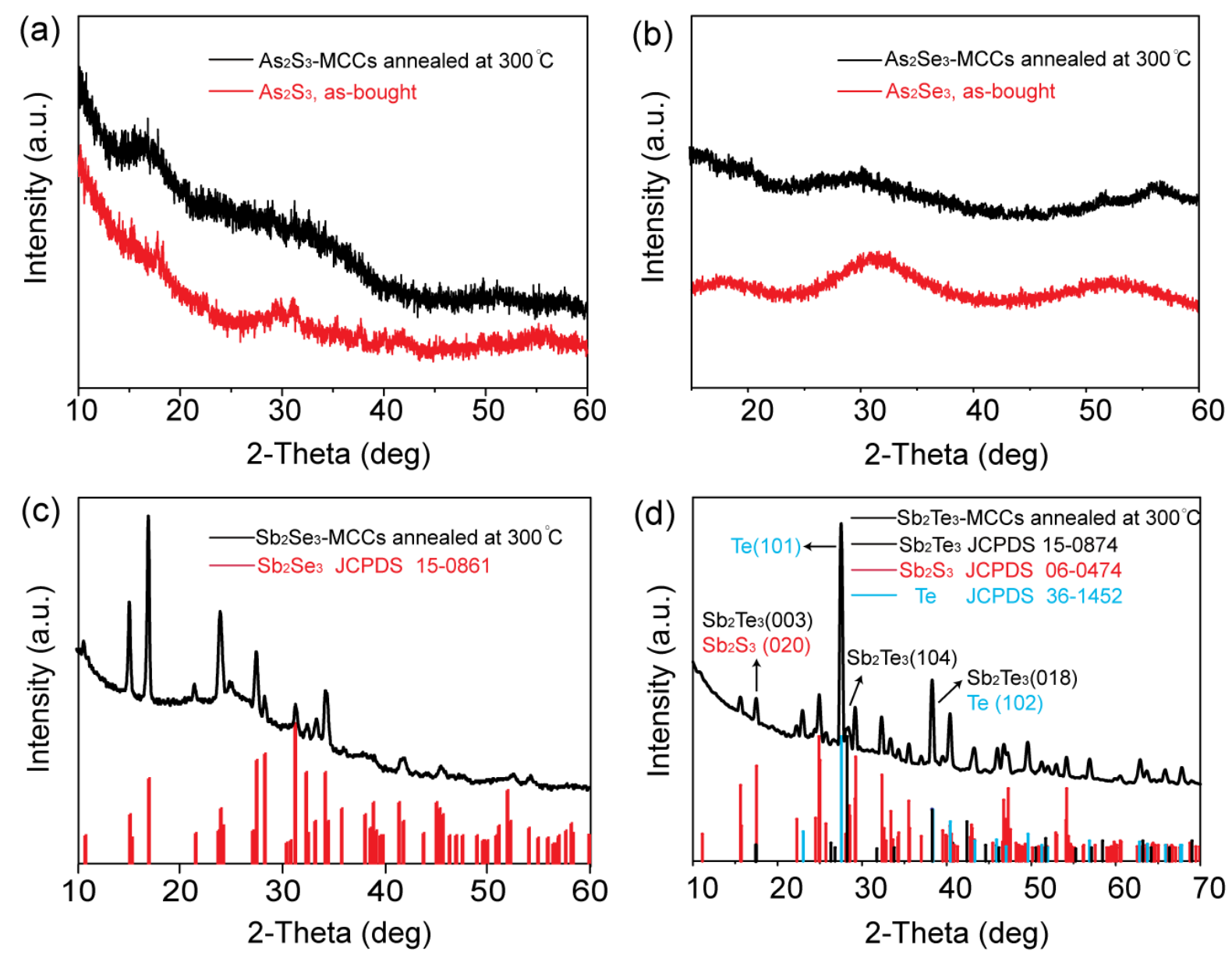

Figure S6. (a) XRD patterns of $\mathrm{As}_{2} \mathrm{~S}_{3}-\mathrm{MCC}$ annealed at $300{ }^{\circ} \mathrm{C}$ (black curve) and as-bought $\mathrm{As}_{2} \mathrm{~S}_{3}$ (red curve). EDS analysis revealed an elemental composition of 42.25 at $\%$ As and 57.75 at $\% \mathrm{~S}$ for as-bought $\mathrm{As}_{2} \mathrm{~S}_{3}$, compared with 41.28 at $\%$ As, and 58.72 at $\% \mathrm{~S}$ for $\mathrm{As}_{2} \mathrm{~S}_{3}$ prepared through thermal decomposition of $\mathrm{As}_{2} \mathrm{~S}_{3}-\mathrm{MCC}$ at $300{ }^{\circ} \mathrm{C}$. (b) XRD patterns of $\mathrm{As}_{2} \mathrm{Se}_{3}-\mathrm{MCC}$ annealed at $300{ }^{\circ} \mathrm{C}$ (black curve) and as-bought $\mathrm{As}_{2} \mathrm{Se}_{3}$ (red curve). EDS analysis revealed an elemental composition of 36.36 at\% As and 63.64 at $\%$ Se for as-bought $\mathrm{As}_{2} \mathrm{Se}_{3}$, compared with 32.90 at $\%$ As, 36.35 at $\%$ Se, and 30.75 at $\% \mathrm{~S}$ for $\mathrm{As}_{2} \mathrm{Se}_{3}$ prepared through thermal decomposition of $\mathrm{As}_{2} \mathrm{Se}_{3}-\mathrm{MCCs}$ at $300{ }^{\circ} \mathrm{C}$. (c) XRD pattern of $\mathrm{Sb}_{2} \mathrm{Se}_{3}-\mathrm{MCC}$ annealed at $300{ }^{\circ} \mathrm{C}$. (d) XRD pattern of $\mathrm{Sb}_{2} \mathrm{Te}_{3}-\mathrm{MCC}$ annealed at $300{ }^{\circ} \mathrm{C}$. 


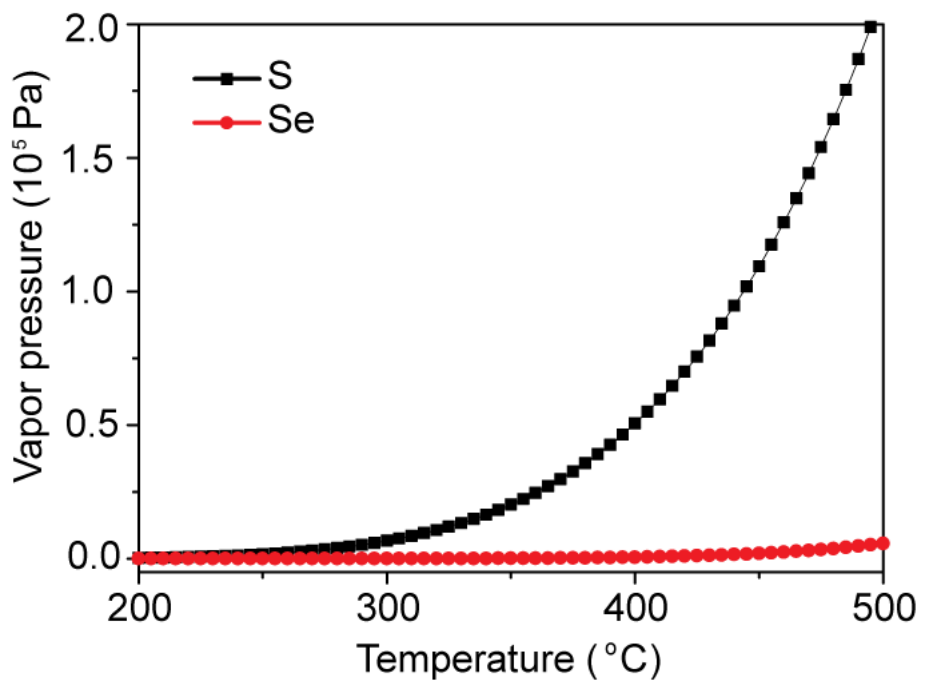

Figure $S_{7}$. Temperature-dependent saturated vapor pressure of $S$ and Se in the temperature range from $200{ }^{\circ} \mathrm{C}$ to $500{ }^{\circ} \mathrm{C}$.

\section{Calculation procedures of Figure $\mathrm{S}_{\mathbf{7}}$ :}

We calculated the temperature dependent vapor pressure of S and Se according to the following Equation:

$$
\log \mathrm{P}(\mathrm{mm} \mathrm{Hg})=\mathrm{A}-\frac{B}{t\left({ }^{\circ} \mathrm{C}\right)+C}
$$

For elemental S, A is 6.84, B is 2500.12 and C is 186.30 ; for elemental Se, A is 7.63, B is 4213.0 and C is 202.0.

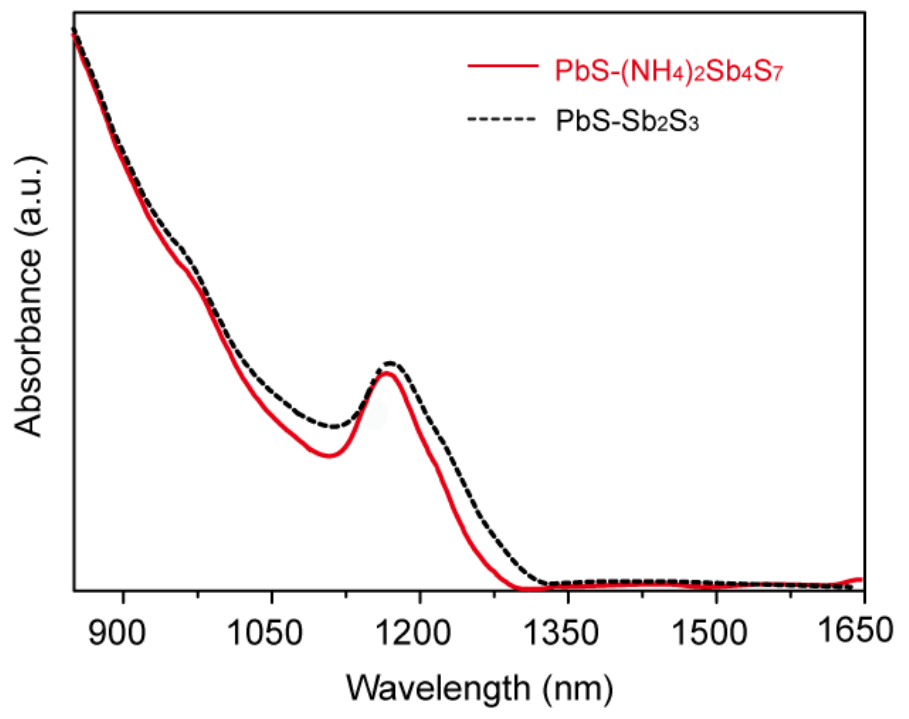

Figure S8. Absorption spectra of $\mathrm{PbS}$ QDs capped with $\left(\mathrm{NH}_{4}\right)_{2} \mathrm{Sb}_{4} \mathrm{~S}_{7}$ ligands before (red line) and after (black line) annealing at $150^{\circ} \mathrm{C}$. 
Table S1. Thermodynamic calculation on Gibbs free energy at 6ooK.

\begin{tabular}{cccccc}
\hline \hline Compounds & $\mathrm{Sb}_{2} \mathrm{O}_{3}(\mathrm{~s})$ & $\mathrm{Sb}_{2} \mathrm{~S}_{3}(\mathrm{~s})$ & $\mathrm{Sb}_{2} \mathrm{Se}_{3}(\mathrm{~s})$ & $\mathrm{H}_{2} \mathrm{~S}(\mathrm{~g})$ & $\mathrm{H}_{2} \mathrm{O}(\mathrm{g})$ \\
\hline $\begin{array}{c}\triangle_{\mathrm{f}} \mathrm{G}_{\mathrm{m}} \\
\mathrm{kJ} \mathrm{mol}{ }^{-1}\end{array}$ & -549.774 & -133.325 & -119.353 & -42.426 & -241.081 \\
\hline \hline $\mathrm{Sb}_{2} \mathrm{O}_{3}(\mathrm{~s})+3 \mathrm{H}_{2} \mathrm{~S}(\mathrm{~g})=\mathrm{Sb}_{2} \mathrm{~S}_{3}(\mathrm{~s})+3 \mathrm{H}_{2} \mathrm{O}(\mathrm{g})$ & $\Delta_{r} G_{m}(600 \mathrm{~K})=-179.516<0$
\end{tabular}

Table S2. Results summary for the composition of $\mathrm{Sb}_{2}\left(\mathrm{~S}_{1-\mathrm{X}} \mathrm{Se}_{\mathrm{x}}\right)_{3}$ films measured by EDS and XRD. Note: EDS results were measured at three randomly selected areas of the sample.

\begin{tabular}{|c|c|c|c|}
\hline NO. & $\begin{array}{c}\text { Composition measured by EDS } \\
\text { (S/Se atom ratio \%) }\end{array}$ & $\begin{array}{c}\text { Composition measured by XRD } \\
\text { (S/Se atom ratio \%) }\end{array}$ & Stoichiometry \\
\hline 1 & 1:0 & $1: 0$ & $\mathrm{Sb}_{2} \mathrm{~S}_{3}$ \\
\hline 2 & $\begin{array}{c}0.5595: 0.4405 \\
0.5481: 0.4519 \\
0.5591: 0.4409\end{array}$ & $0.5581: 0.4419$ & $\mathrm{Sb}_{2}\left(\mathrm{~S}_{0.56} \mathrm{Se}_{0.44}\right)_{3}$ \\
\hline 3 & $\begin{array}{c}0.5447: 0.4553 \\
0.5211: 0.4789 \\
0.5215: 0.4785\end{array}$ & $0.5181: 0.4819$ & $\mathrm{Sb}_{2}\left(\mathrm{~S}_{0.52} \mathrm{Se}_{0.48}\right)_{3}$ \\
\hline 4 & $\begin{array}{l}0.4402: 0.5598 \\
0.4586: 0.5414 \\
0.4648: 0.5352 \\
\end{array}$ & $0.4374: 0.5626$ & $\mathrm{Sb}_{2}\left(\mathrm{~S}_{\mathrm{o} .44} \mathrm{Se}_{\mathrm{o} .56}\right)_{3}$ \\
\hline 5 & $\begin{array}{c}0.2429: 0.7571 \\
0.2274: 0.7726 \\
0.2573: 0.7427\end{array}$ & o.2447:0.7553 & $\mathrm{Sb}_{2}\left(\mathrm{~S}_{0.24} \mathrm{Se}_{0.76}\right)_{3}$ \\
\hline 6 & o:1 & o:1 & $\mathrm{Sb}_{2} \mathrm{Se}_{3}$ \\
\hline
\end{tabular}

Table S3. Device performance parameters of the $\mathrm{TiO}_{2} / \mathrm{Sb}_{2}\left(\mathrm{~S}_{0.44} \mathrm{Se}_{0.56}\right)_{3}$ thin film solar cell include the best and the average parameters (with standard deviation). Thirty devices were analyzed for the statistics.

\begin{tabular}{|c|c|c|c|c|c|c|c|}
\hline $\begin{array}{l}\text { Voc } \\
(\mathrm{V})\end{array}$ & $\begin{array}{c}\text { Jsc } \\
\left(\mathrm{mA} \mathrm{cm}^{-2}\right)\end{array}$ & $\begin{array}{l}\text { FF } \\
(\%)\end{array}$ & $\begin{array}{r}\text { PCE } \\
(\%)\end{array}$ & $\begin{array}{l}\text { Rs } \\
(\Omega)\end{array}$ & $\begin{array}{l}\text { Rsh } \\
(\Omega)\end{array}$ & A & $\begin{array}{c}\mathrm{J}_{\mathrm{o}} \\
\left(\mathrm{mA} \mathrm{cm}^{-2}\right)\end{array}$ \\
\hline $\begin{array}{c}0.49 \\
(0.47 \pm 0.02)\end{array}$ & $\begin{array}{c}6.6 \\
(6.7 \pm 0.2)\end{array}$ & $\begin{array}{c}44.2 \\
(41.6 \pm 2.2)\end{array}$ & $\begin{array}{c}1.43 \\
(1.33 \pm 0.08)\end{array}$ & $\begin{array}{c}229 \\
(255 \pm 24)\end{array}$ & $\begin{array}{c}2457 \\
(2132 \pm 341)\end{array}$ & 2.46 & $2.0 \times 10^{-2}$ \\
\hline
\end{tabular}

